\title{
ELECTRON FIELD EMISSION STUDIES OF THE OXIDATION OF NICKEL
}

\author{
A qualitative study of oxygen adsorption on clean nickel surfaces \\ made by the National Bureau of Standards.
}

An electron field emission microscope has been applied to a qualitative study of oxygen adsorption on nickel. The study was made by W. J. Ambs of the National Bureau of Standards (NBS), US Dept. of Commerce, as part of a program of research into fundamental corrosion reactions at metal surfaces. ${ }^{1}$ It was sponsored in part by the Advanced Research Projects Agency.

During oxygen adsorption, the nickel emission patterns developed sharp bright rings around the (110) crystallographic planes, indicating that a rapid rearrangement of atoms was taking place in those areas. This observation was interpreted as a confirmation of similar results obtained elsewhere in low-energy electron diffraction experiments with oxygen adsorption on nickel. ${ }^{2}$ Some preliminary field emission work with iron was also performed during the NBS study.

Studies of the growth of oxide films on metals show that details of the formation of the first few molecular layers are crucial in determining the subsequent course of the oxidation. These details are impossible to observe unless the metallic surface is completely clean and its atomic geometry reasonably well-known. Using the electron field emission microscope, the observer can establish procedures to ensure cleanliness of the initial metallic surface, and can observe all facets of the crystal simultaneously at high magnification.

Basically, a field emission microscope consists of a needle-shaped electrically conducting emitter (usually made of a metal) and a phosphor screen within an evacuated Pyrex tube. A difference in potential between the emitter and the screen is maintained at a value sufficiently large to cause electrons to leave the surface of the metal by quantum mechanical tunneling and then to accelerate them to the screen. The emitter tip is hemispherically shaped so that electrons are emitted radially to form a magnified image of the tip surface on the screen. Local variations of radius of curvature and of electron emission barrier (work function) at the metal surface produce the contrast necessary for identification of crystallographic planes.
In principle, the field emission microscope presents a direct method of observing the events occurring on a metallic surface. Yet, although available for about 25 years, it has seldom been applied to the study of metal oxidation; in most oxidation work a measure of oxide film thickness as a function of time is desired, and this is the one thing the field emission microscope fails to provide.

However, the instrument does give an approximation to a stereographic map of the work function distribution of a hemispherical crystal surface. Hence, it usually reveals the presence of epitaxial layers, foreign particles, and, under certain conditions, perhaps even individual adsorbed molecules.

All changes which involve local alteration of work function or local change in tip radius of the emitter cause a change in the appearance of the pattern. Since the useful magnification of the instrument is comparable with that of the conventional electron microscope, the field emission microscope reveals events on an almost atomic scale, making it useful in the study of nucleation phenomena. In only a relatively small number of the investigations reported has advantage been taken of this highly useful magnification.

Most field emission studies of metal oxidation have been confined to such refractory metals as tungsten, molybdenum, and tantalum because their oxides can easily be removed by vaporization within an evacuated system. Of much greater interest chemically, however, are such transition metals as nickel, iron, and platinum, which act as catalysts in many reactions. The oxides that normally form on these elements are difficult to remove. Vaporization cannot be used for some of these metals because the melting points of the metals are too close to the temperatures at which their oxides vaporize. Nickel, however, has a melting temperature of $1455^{\circ} \mathrm{C}$, while its oxide vaporizes at about $1040^{\circ} \mathrm{C}$. With great care, therefore, nickel can be studied with field emission.

One objective of the NBS study was to observe the nickel-oxygen reaction in greater detail than had previous investigators elsewhere. For this reason, the oxygen pres- sures used were lower than in the earlier studies. The oxygen was introduced through a silver diffusionthimble that, when heated to about $650^{\circ} \mathrm{C}$, permits oxygen to diffuse into the system.

At an oxygen pressure of $10^{-8}$ torr and room temperature, the intensity of the emission pattern slowly decreased as oxygen was adsorbed on all planes. Simultaneously, small sharply defined, bright rings centered on the (110) planes developed. These rings differ considerably from those observed around the (110) and (100) planes at higher pressures, ${ }^{3}$ and therefore represent strong support of findings by the electron-diffraction method. ${ }^{2}$

Related experiments undertaken at the NBS to substantiate this confirmation showed that the rings are not due to carbon dioxide or other gases formed in the ion gauge and that the adsorption process is distinct from the surface rearrangement, which is thermally activated. This manifestation of the adsorption process is easily missed if the surface is at all contaminated.

Also of interest is the nucleation of crystallites on the nickel surface during oxidation. When a clean nickel tip was heated to about $600^{\circ} \mathrm{C}$ with oxygen pressure of $10^{-6}$ torr, both bright and dark crystallites were observed. However, on a tip treated with oxygen at room temperature and $10^{-8}$ torr and then heated to about $500^{\circ}$ to $600^{\circ} \mathrm{C}$ at $10^{-9}$ torr, only bright crystallites were found.

The crystallites are more prominent if the nickel contains dissolved nickel oxide. The differences in emission may be due to differences in stoichiometry of the crystals growing on different faces. Such variations in stoichiometry could easily influence the electrical properties of the crystals.

In the preliminary experiments on iron, the phase transition at $910^{\circ} \mathrm{C}$ was observed. Performance of the experiment was difficult, and observation of this transition is considered remarkable because of the structural weakness of pure iron at this temperature and the extremely heavy stress placed on the emitter by the electric field.

In preliminary oxidation experiments on iron whiskers, a preferential darkening of the (111) planes was noted. It was also found that iron whiskers can be grown by heating iron field emitters to about $900^{\circ} \mathrm{C}$ in the electric field.

\section{References}

1 For further details, see Field-Emission Studies of the Oxidation of Clean Nicke Surfaces, by William J. Ambs, Proc. 2nd International Congress on Metallic Corrosion, March 1963, to be published.

${ }^{2}$ L. H. Germer and A. U. MacRae: Welch Found. Research Bull., vol. 11, 1961.

${ }^{3}$ R. Gomer: J. Chem. Phys., 1953, p. 293. 